\title{
Synthesis and Reactions of Some Uracil and 5-Halouracil Nucleosides of 2-Acetamido-2-deoxy- D-glucose
}

\author{
Najim A. Al-Masoudi* and Fadhel B. Issa \\ Department of Chemistry, College of Science, University of Basrah, Basrah, Iraq
}

\begin{abstract}
Al-Masoudi, N. A. and Issa, F. B., 1997. Synthesis and Reactions of Some Uracil and 5-Halouracil Nucleosides of 2-Acetamido-2-deoxy-D-glucose. - Acta Chem. Scand. 51: 958-962. (C) Acta Chemica Scandinavica 1997.

Silylated uracils react with 2 -acetamido-1,3,4,6-tetra- $O$-acetyl-2-deoxy-D-glucose (5) under trimethylsilyl trifluoromethanesulfonate catalysis to give the nucleosides 6, 8, 10 and 12, respectively, as well as the diglycoside 14. Deblocking with sodium methoxide in methanol afforded the free nucleosides 7, 9, 11 and 13 respectively. Alternatively, the free nucleosides $\mathbf{1 1}$ and $\mathbf{1 3}$ were obtained directly by halogenation of 7 with $\mathrm{N}$-bromosuccinimide or iodine monochloride, respectively. The structures of the synthesized compounds were confirmed by their ${ }^{1} \mathrm{H}$ NMR, UV and mass spectra and elemental analyses.
\end{abstract}

Modified nucleosides have been used among other compounds as broad spectrum antiviral, antibacterial or antitumor agents. ${ }^{1}$ Several 5-halouracil nucleosides of chemical and biochemical interest ${ }^{2}$ have been investigated extensively as antineoplastic and antiviral agents. ${ }^{3}$ Some 5-chlorouracil nucleosides, which are less cytotoxic, exhibit selective anti-HIV activity. ${ }^{4}$ 5-Fluoro-2'-deoxyuridine exhibits antileukemic activity, while its 5 '-phosphate (FDUMP) is a potent inhibitor of the enzyme thymidylate synthase, and an active antitumor and antifungal agent. ${ }^{5-7}$ On the other hand, 5-fluoro-2-thio-2'-deoxyuridine ( $\mathrm{S}^{4} \mathrm{FDUMP}$ ) and its 2-thiocytosine analogue ${ }^{8}$ proved to be effective inhibitors of proliferation of several mammalian tumor cell lines. ${ }^{9}$ Ollapally et al. ${ }^{10}$ have recently reported antiviral and in vitro antineoplastic activities of some keto unsaturated nucleosides of L-rhamnose carrying 6-azauracil and 5-fluorouracil. Some iodo analogues have been proved to be effective against viral infections, for example 5-iodo-2'-deoxyuridine (IDDU $)^{11}$ and the new, promising antiherpetic agent $\left(2 S^{\prime}\right)-2^{\prime}$-methyl-5-iodouridine (SMIU) ${ }^{12}$ In addition, halogenated nucleosides have also been used as synthons for the synthesis of many biologically active nucleosides. ${ }^{13-18}$ We report now the synthesis of a new type of 5-halouracil nucleosides carrying a 2-acetamido-2-deoxyD-glucosyl moiety at $\mathrm{N}-1$ as promising antiviral, antimutagenic, antineoplastic agents or enzyme inhibitors. There is broad biochemical interest in D-glucosamine ${ }^{19-21}$ and 5-fluorouracil itself possesses antileukemic properties. ${ }^{22}$

* To whom correspondence should be addressed.

\section{Result and discussion}

In recent years, Stevens et al. ${ }^{23}$ described the chemical synthesis, physical properties and antitumor activity of 1-(2-acetamido-2-deoxy- $\beta$-D-glucopyranosyl) uracil (7). This compound was prepared from glycosylation of 1 chloro-2-acetamido-3,4,6-tri- $O$-acetyl-2-deoxy-D-glucose with 2,4-dimethoxypyrimidine according to the HilbertJohnson procedure ${ }^{24}$ followed by deprotection under acidic conditions. The structural assignment of the $\beta$-configuration of 7 was based on its specific rotation only. In the present work, the nucleoside 7 is proposed as a useful intermediate for the synthesis of some potentially biological active 5-halouracil nucleosides. The easy accessibility and the biochemical interest of 2-acetamido2-deoxy-D-glucose prompted us to use this aminosugar as a starting material in glycosylation reactions of the Hilbert-Johnson-Birkofer type. ${ }^{25}$ Thus, trimethylsilylated derivatives of uracil, 5-fluorouracil, 5-bromouracil and 5-iodouracil (1-4), respectively, were condensed with 2-acetamido-1,3,4,6-tetra- $O$-acetyl-2-deoxy-D-glucose (5) in the presence of trimethylsilyl triflate as catalyst ${ }^{26}$ in dry 1,2-dichloroethane. The reaction proceeded at boiling temperature to give the desired 1-(2-acetamido-3,4,6-tri- $O$-acetyl-2-deoxy- $\beta$-D-glucopyranosyl) uracil (6) and its 5-halo analogues 8, 10 and 12 in yields of $45,36,48$ and $45 \%$, respectively. These reactions are thermodynamically controlled and form, by neighboring group participation of the 2-acetamido group, almost exclusively the $\beta$-anomers. Deacetylation proceeded smoothly with sodium methoxide in dry methanol at room temperature to give the known free 


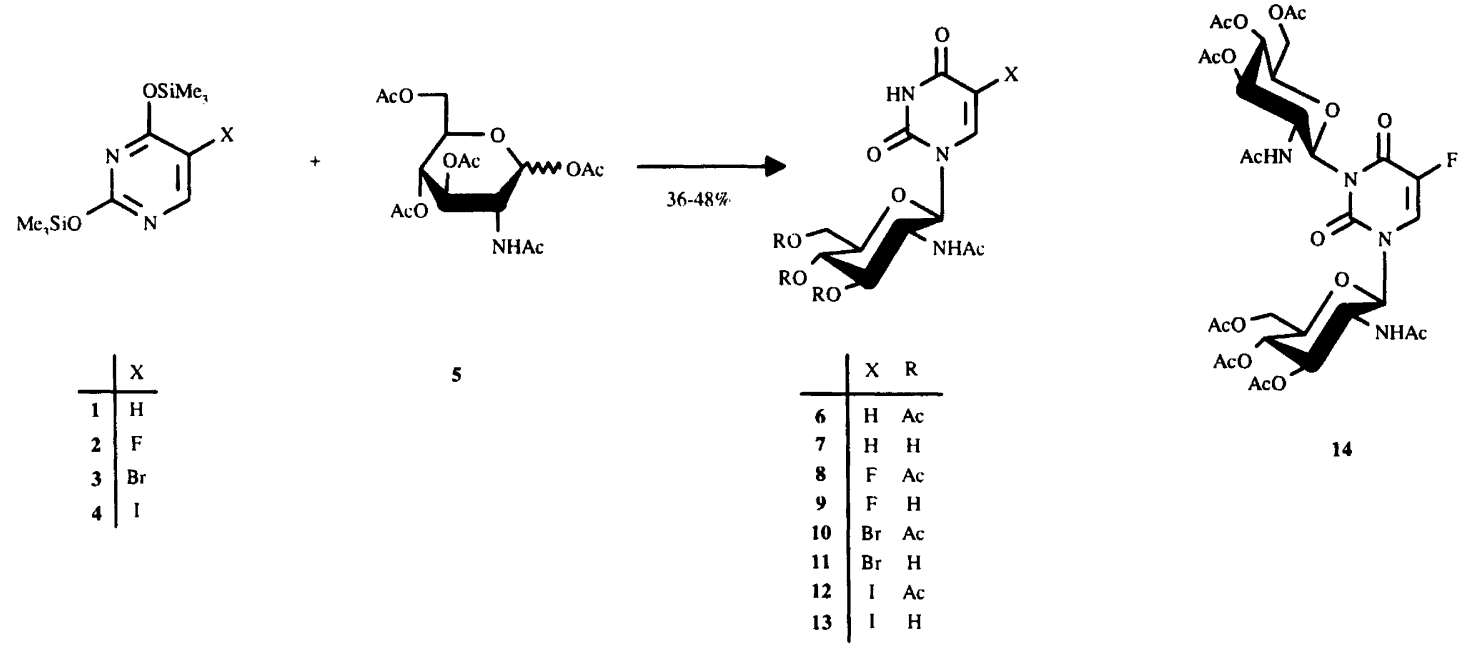

Scheme 1.

nucleoside 7 (76\% yield), ${ }^{23} 9(63 \%), 11(60 \%)$ and 13 (77\%). The diglycoside 14 was obtained, in addition to the monoglycoside $\mathbf{8}$, from the reaction of the silylated 5 -fluorouracil with $\mathbf{5}$, as a minor component in $5 \%$ yield (Scheme 1).

An attempt to synthesize the free 5-halonucleosides from the parent uracil derivative 7 , using the method of Knaus et al. ${ }^{27}$ has been examined. The reaction of 7 with $\mathrm{N}$-bromosuccinimide or iodine monochloride, in the presence of sodium azide at 35 and $25^{\circ} \mathrm{C}$, gave the 5bromo- and 5-iodo-uracil nucleosides $\mathbf{1 1}$ and $\mathbf{1 3}$ in 56 and $50 \%$ yield, respectively. The formation of these products was assumed to occur via a 5-halo-6-azido-5,6dihydro intermediate, followed by elimination of $\mathrm{HN}_{3}$ (Scheme 2).

The structures of the synthesized uracil nucleosides were determined on the basis of their $\mathrm{UV},{ }^{1} \mathrm{H} \mathrm{NMR},{ }^{13} \mathrm{C}$ NMR and mass spectra which were found to be consistent with the assigned structures. The formation of $\mathrm{N}-1$ nucleosides was confirmed by UV spectral comparison with known structures of glycopyranoside analogues and 1 (5-thio- $\beta$-D-xylopyranosyl) pyrimidines. ${ }^{28}$

The ${ }^{1} \mathrm{H}$ NMR spectra of $6,8,10$ and 12 showed the anomeric protons as doublets at $\delta 5.64,5.71,5.74$ and 5.40 with $J$ coupling of $9.5,9.2,9.5$ and $10.0 \mathrm{~Hz}$, respectively, corresponding to a diaxial orientation of $\mathrm{H}-1^{\prime}$ and
$\mathrm{H}-2^{\prime}$ protons which are indicative of the $\beta$-configuration. The large coupling constants $J_{2^{\prime}, 3^{\prime}}, J_{3^{\prime}, 4^{\prime}}$ and $J_{4^{\prime}, 5^{\prime}}$ $(9.0-10.0 \mathrm{~Hz})$ confirmed the ${ }^{4} C_{1}$ conformation of the sugar moiety. The four acetyl groups appeared as three singlets at $\delta 1.92-2.08$, while signals appearing at $\delta$ 1.68-1.94 were attributed to the acetamido groups. Similarly, the free nucleosides 7, 9, 11 and 13 showed, in their ${ }^{1} \mathrm{H}$ NMR spectra, the signals from anomeric protons as doublets at $\delta 5.57,5.355 .39$ and $5.38,5.57$ with large $J_{1^{\prime}, 2^{\prime}}$ coupling constants $(10.0,9.7,9.5$ and 9.5 , respectively) clearly indicating that these compounds have also the $\beta$-configuration. The assignments of the hydroxy groups in these compounds were determined from $\mathrm{D}_{2} \mathrm{O}$ exchange. The ${ }^{1} \mathrm{H}$ NMR spectrum of 14 showed large $J_{\mathrm{H}, \mathrm{H}}$ coulpings $\left[J_{\left(1^{\prime}, 2^{\prime}\right) \mathrm{a}}, J_{\left(1^{\prime}, 2^{\prime}\right) \mathrm{b}} 9.5 \mathrm{~Hz} ; J_{\left(4^{\prime}, 5^{\prime}\right) \mathrm{a}}, J_{\left(4^{\prime}, 5^{\prime}\right) \mathrm{b}}\right.$ $9.5 \mathrm{~Hz}$ ] indicative of the $\beta$-form with the ${ }^{4} C_{1}$ conformation of the sugar moieties at $\mathrm{N}-1$ and $\mathrm{N}-3$. In the ${ }^{13} \mathrm{C}$ NMR spectrum of 14 the signals at $\delta$ 171.6-169.2 were attributed to the ester carbonyl carbon atoms, while that at $\delta 148.2$, to the C-5 atom of the nucleobase. A signal at $\delta 124.1$ was assigned to the $\mathrm{C}-6$ atom. The anomeric carbon atoms $\mathrm{C}-1_{\mathrm{a}}^{\prime}$ and $\mathrm{C}-\mathrm{l}_{\mathrm{b}}^{\prime}$ appeared at $\delta 81.2$ and 80.8, respectively. Another ten signals at $\delta$ 75.1-49.5 were assigned to $\mathrm{C}-2^{\prime}, \mathrm{C}-3^{\prime}, \mathrm{C}-4^{\prime}, \mathrm{C}-5^{\prime}$ and $\mathrm{C}-6^{\prime}$ of both sugar moieties at $\mathrm{N}-1$ and $\mathrm{N}-3$, while signals at $\delta$ 23.2-20.5 were attributed to the acetoxy methyl carbons.

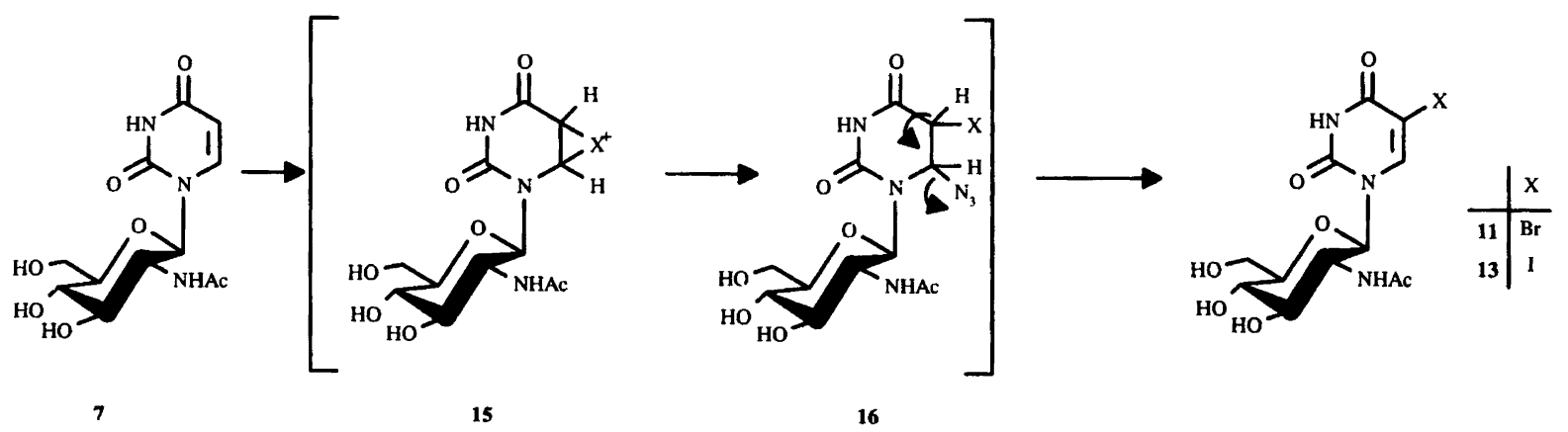

Scheme 2. 
In summary, we have achieved a regiospecific synthesis of 5-halouracil nucleosides by the direct glycosylation of 5-halouracil with peracylated D-glucosamine and via the halogenation of the uracil nucleoside 7 by an electrophilic ha!ogen reagent and sodium azide under mild conditions. These nucleosides will be utilized for the biological evaluation studies.

\section{Experimental}

General. ${ }^{1} \mathrm{H}$ NMR and ${ }^{13} \mathrm{C}$ NMR spectra were measured with Bruker WM 250 and Bruker AC 250 spectrometers (unless otherwise stated) with tetramethylsilane as an internal standard and on a $\delta$ scale in ppm. UV spectra were recorded on a Perkin-Elmer spectrophotometer Lambda 5. Thin layer chromatography was performed on silica gel sheets F 1550 LS 254 from Scheicher \& Schüll. Silica gel 60 (Merck) was used for column chromatography. EI and FAB mass spectra were recorded on a MAT 312 mass spectrometer using 3-nitrophenol $(\mathrm{NBOH})$ or glycerol as matrix. Some molecular ions were detected by doping the samples with $\mathrm{Na}^{+}$ion. The melting points were determined with a Dr. Tottli melting point apparatus and are not corrected.

5-Halouracil nucleosides of 2-acetamido-3,4,6-tri-Oacetyl-2-deoxy- $\beta$-D-glucose (6), (8), (10) and (12). General method. A suspension of 5-fluoro-, 5-bromo- or 5-iodo-uracils $(3.84 \mathrm{mmol})$ in hexamethyldisilazane $(20 \mathrm{ml})$ containing a few crystals of $\left(\mathrm{NH}_{4}\right)_{2} \mathrm{SO}_{4}$ was heated under reflux for $8 \mathrm{~h}$. After cooling, the solutions were evaporated to dryness to give crude 1-4. These were dissolved in dry 1,2-dichloroethane $(20 \mathrm{ml})$, and a solution of the sugar 5 (3.84 mmol) was added, followed by trimethylsilyl trifilate catalyst $(0.70 \mathrm{mmol})$. The solutions were heated under reflux for $3 \mathrm{~h}$ and then cooled and evaporated to dryness. The residues were chromatographed on silica gel $(50 \mathrm{~g})$ using a gradient of $\mathrm{MeOH}$ $(0-0.5 \%)$ in $\mathrm{CHCl}_{3}$.

1 - (2-Acetamido-3,4,6-tri-O-acetyl-2-deoxy- $\beta$-D-glucopyranosyl)uracil (6). Yield: $0.76 \mathrm{~g} \quad(45 \%) ; \quad R_{\mathrm{f}}$ $\left(\mathrm{CHCl}_{3}-\mathrm{MeOH}\right.$ 9:1) 0.48, m.p. $161-165^{\circ} \mathrm{C}$ decomp. (from EtOAc). UV: $\lambda_{\max }(\mathrm{MeOH}) 273(\log \varepsilon 4.06) .{ }^{1} \mathrm{H}$ NMR: $\delta 11.43$ (s,1 H, NH), 8.08 (d, $1 \mathrm{H}, \mathrm{H}-6), 7.89$ (d, $\left.1 \mathrm{H}, \mathrm{NHAc}, J_{2^{\prime}, \mathrm{NH}} 7.7 \mathrm{~Hz}\right), 5.76\left(\mathrm{~d}, 1 \mathrm{H}, \mathrm{H}-5, J_{5,6} 7.8 \mathrm{~Hz}\right)$, $5.64\left(\mathrm{~d}, 1 \mathrm{H}, \mathrm{H}-1^{\prime}, J_{1^{\prime}, 2^{\prime}} 9.5 \mathrm{~Hz}\right), 5.24\left(\mathrm{t}, 1 \mathrm{H}, \mathrm{H}-3^{\prime}, J_{3^{\prime}, 4^{\prime}}\right.$ $9.8 \mathrm{~Hz}), 5.12\left(\mathrm{dd}, 1 \mathrm{H}, \mathrm{H}-4^{\prime}, J_{4^{\prime}, 5^{\prime}} 9.0 \mathrm{~Hz}\right), 4.51(\mathrm{q}, 1 \mathrm{H}$, H-2', $\left.J_{2^{\prime}, 3^{\prime}} 9.8 \mathrm{~Hz}\right), 4.20-3.89$ (m, $3 \mathrm{H}, \mathrm{H}-5^{\prime}, \mathrm{H}-6^{\prime}, \mathrm{H}-6^{\prime \prime}$ ), 2.01, 1.99, 1.93 ( $3 \mathrm{~s}, 9 \mathrm{H}, 3 \mathrm{OAc}), 1.77$ (s, $3 \mathrm{H}, \mathrm{NHAc}$ ). Found: C 47.75; $\mathrm{H}$ 5.19; N 9.81. Calc. for $\mathrm{C}_{18} \mathrm{H}_{23} \mathrm{~N}_{3} \mathrm{O}_{10}$ : C 48.00; H 5.30; N 9.50; MS: $m / z(\mathrm{EI}>0) 442(M+\mathrm{H})^{+}$.

1 - (Acetamido-3,4,6 - tri-O-acetyl-2 - deoxy- $\beta$ - D-glucopyranosyl)-5-fluorouracil (8). Yield: $0.68 \mathrm{~g}(36 \%) ; R_{\mathrm{f}}$ ( $\left.\mathrm{CHCl}_{3}-\mathrm{MeOH} 9: 1\right)$ 0.34, m.p. $115-120^{\circ} \mathrm{C}$, decomp. at $205^{\circ} \mathrm{C}$. UV: $\lambda_{\max }(\mathrm{MeOH}) 263(\log \varepsilon 3.86) .{ }^{1} \mathrm{H}$ NMR: $\delta 11.88(\mathrm{~s}, 1 \mathrm{H}, \mathrm{NH}), 8.36\left(\mathrm{~s}, 1 \mathrm{H}, \mathrm{H}-6, J_{6, \mathrm{~F}} 7.3 \mathrm{~Hz}\right.$ ), $8.10\left(\mathrm{~d}, 1 \mathrm{H}, \mathrm{NHAc}, J_{2^{\prime}, \mathrm{NH}} 9.3 \mathrm{~Hz}\right), 5.71\left(\mathrm{~d}, 1 \mathrm{H}, \mathrm{H}-1^{\prime}\right.$, $\left.J_{1^{\prime}, 2^{\prime}} 9.2 \mathrm{~Hz}\right), 5.24\left(\mathrm{t}, 1 \mathrm{H}, \mathrm{H} 3^{\prime}, J_{3^{\prime}, 4^{\prime}} 9.6 \mathrm{~Hz}\right), 5.12(\mathrm{t}$, $\left.1 \mathrm{H}, \mathrm{H}-4^{\prime}, J_{4^{\prime}, 5^{\prime}} 9.4 \mathrm{~Hz}\right), 4.25$ (q, $1 \mathrm{H}, \mathrm{H}-2^{\prime}, J_{2^{\prime}, 3^{\prime}} 9.7 \mathrm{~Hz}$ ), 4.06-3.31 (m, 3 H, H-5', H-6', H-6"), 2.00, 1.97, 1.92 ( $3 \mathrm{~s}, 9 \mathrm{H}, 3 \mathrm{OAc}$ ), 1.71 (s, $3 \mathrm{H}, \mathrm{NH} \underline{\mathrm{Ac}}$ ). Found: C 46.86; $\mathrm{H}$ 4.78; $\mathrm{N}$ 9.29. Calc. for $\mathrm{C}_{18} \mathrm{H}_{22} \mathrm{FN}_{3} \mathrm{O}_{10}$ : C 47.06; $\mathrm{H}$ 4.83 ; N 9.15 .

1,3-Bis (2-acetamido-3,4,6-tri-O-acetyl-2-deoxy- $\beta$-D-glucopyranosyl)-5-fluorouracil (14). Yield: $0.15 \mathrm{~g}(5 \%) ; R_{\mathrm{f}}$ ( $\mathrm{CHCl}_{3}-\mathrm{MeOH}$ 9:1) 0.62, m.p. 204-206 ${ }^{\circ} \mathrm{C}$ (decomp.). UV: $\lambda_{\max }(\mathrm{MeOH}) 268 \quad(\log \varepsilon \quad 3.82) .{ }^{1} \mathrm{H} \quad \mathrm{NMR}$ $(600 \mathrm{MHz}): \delta 7.45\left(\mathrm{~d}, 1 \mathrm{H}, \mathrm{H}-6, J_{6, \mathrm{~F}} 5.5 \mathrm{~Hz}\right), 6.18(\mathrm{~d}$, $\left.1 \mathrm{H}, \mathrm{NHAc}, J_{2^{\prime}, \mathrm{NH}} 9.2 \mathrm{~Hz}\right), 5.90\left(\mathrm{~d}, 1 \mathrm{H}, \mathrm{NHAc}, J_{2^{\prime}, \mathrm{NH}}\right.$ $9.0 \mathrm{~Hz}), 5.87\left(\mathrm{~d}, 1 \mathrm{H}, \mathrm{H}-1^{\prime}, J_{1^{\prime}, 2^{\prime}} 9.7 \mathrm{~Hz}\right), 5.81(\mathrm{~d}, 1 \mathrm{H}$, $\left.\mathrm{H}-1^{\prime}, J_{1^{\prime}, 2^{\prime}} 9.5 \mathrm{~Hz}\right), 5.27\left(\mathrm{t}, 1 \mathrm{H}, \mathrm{H}-3^{\prime}, J_{3^{\prime}, 4^{\prime}} 9.7 \mathrm{~Hz}\right), 5.17$ $\left(\mathrm{t}, 1 \mathrm{H}, \mathrm{H}-3^{\prime}, J_{3^{\prime}, 4^{\prime}} 9.5 \mathrm{~Hz}\right), 5.13\left(\mathrm{t}, 1 \mathrm{H}, \mathrm{H}^{\prime} 4^{\prime}, J_{4^{\prime}, 5^{\prime}}\right.$ $9.7 \mathrm{~Hz}), 5.07\left(\mathrm{t}, 1 \mathrm{H}, \mathrm{H}-4^{\prime}, J_{4^{\prime}, 5^{\prime}} 9.5 \mathrm{~Hz}\right), 4.30(\mathrm{q}, 1 \mathrm{H}$, $\left.\mathrm{H}-2^{\prime}, J_{2^{\prime}, 3^{\prime}} 9.7 \mathrm{~Hz}\right), 4.21$ (q, H-2', $\left.J_{2^{\prime}, 3^{\prime}} 9.5 \mathrm{~Hz}\right), 4.19$ (dd, $1 \mathrm{H}, \mathrm{H}-6^{\prime}, J_{5^{\prime}, 6^{\prime \prime}} 2.0 \mathrm{~Hz}$ ), 4.18 (dd, $1 \mathrm{H}, \mathrm{H}-6^{\prime \prime}, J_{6^{\prime}, 6^{\prime \prime}}$ $2.0 \mathrm{~Hz}$ ), 4.17 (dd, $\left.1 \mathrm{H}, \mathrm{H}-6^{\prime}, J_{5^{\prime}, 6^{\prime \prime}} 2.0 \mathrm{~Hz}\right), 4.10$ (dd, $1 \mathrm{H}$, H-6", $J_{6^{\prime}, 6^{\prime \prime}} 12.0 \mathrm{~Hz}$ ), 3.86 (ddd, $1 \mathrm{H}, \mathrm{H}-5^{\prime}, J_{5^{\prime}, 6^{\prime}} 4.2 \mathrm{~Hz}$ ), 3.77 (ddd, $1 \mathrm{H}, \mathrm{H}-5^{\prime}, J_{5^{\prime}} .6^{\prime} 4.0 \mathrm{~Hz}$ ), 2.09, 2.08, 2.07, 2.04, $2.03,2.02(6 \mathrm{~s}, 18 \mathrm{H}, 6 \mathrm{OAc}), 1.94,1.77(2 \mathrm{~s}, 6 \mathrm{H}$, 2 NH스).${ }^{13} \mathrm{C}$ NMR: $\delta 171.6,171.5,171.4,171.3,170.7$, $170.6(2), 169.2(\mathrm{C}=\mathrm{O}), 148.2(\mathrm{C}-5), 124.1(\mathrm{C}-6), 81.2$ (C-1'), $80.8\left({\left.\mathrm{C}-1{ }^{\prime}\right),}^{\prime} 75.1\right.$ (C-2'), $75.0\left(\mathrm{C}-2^{\prime}\right), 73.2\left(\mathrm{C}-3^{\prime}\right)$, 73.1 (C-3'), $67.6\left(\mathrm{C}-4^{\prime}\right), 67.5\left(\mathrm{C}-4^{\prime}\right), 62.1\left(\mathrm{C}-6^{\prime}\right), 61.6$ $\left(\mathrm{C}^{\prime} 6^{\prime}\right), 51.6\left(\mathrm{C}-5^{\prime}\right), 49.5\left(\mathrm{C}-5^{\prime}\right)$. Found: C 48.51; H 5.12; $\mathrm{N}$ 7.02. Calc. for $\mathrm{C}_{32} \mathrm{H}_{41} \mathrm{FN}_{4} \mathrm{O}_{18}$ : C 48.73; $\mathrm{H} \mathrm{5.24;} \mathrm{N}$ 7.10. MS: $m / z(\mathrm{EI}<0) 668\left(M^{+}-2 \mathrm{HOAc}\right)$.

1 - (2-Acetamido-3,4,6-tri-O-acetyl-2-deoxy- $\beta$-D-glucopyranosyl)-5-bromouracil (10). Yield: $0.96 \mathrm{~g},(48 \%) ; R_{\mathrm{f}}$ ( $\mathrm{CHCl}_{3}-\mathrm{MeOH}$ 9:1) 0.41, m.p. 255-258 ${ }^{\circ} \mathrm{C}$ (decomp.). (from EtOAc). UV: $\lambda_{\max }(\mathrm{MeOH}) 273(\log \varepsilon 4.06) .{ }^{1} \mathrm{H}$ NMR: $\delta 8.47$ (s, $1 \mathrm{H}, \mathrm{H}-6), 8.03\left(\mathrm{~d}, 1 \mathrm{H}, \mathrm{NHAc}, J_{2^{\prime}, \mathrm{NH}}\right.$ $9.5 \mathrm{~Hz}), 5.74\left(\mathrm{~d}, 1 \mathrm{H}, \mathrm{H}-1^{\prime}, J_{1^{\prime}, 2^{\prime}} 9.5 \mathrm{~Hz}\right), 5.25(\mathrm{t}, 1 \mathrm{H}$, $\left.\mathrm{H}-3^{\prime}, J_{3^{\prime}, 4^{\prime}} 9.5 \mathrm{~Hz}\right), 5.18\left(\mathrm{t}, 1 \mathrm{H}, \mathrm{H}-4^{\prime}, J_{4^{\prime}, 5^{\prime}} 9.5 \mathrm{~Hz}\right), 4.53$ $\left(\mathrm{q}, 1 \mathrm{H}, \mathrm{H}-2^{\prime}, J_{2^{\prime}, 3^{\prime}} 9.0 \mathrm{~Hz}\right), 4.02\left(\mathrm{~m}, 3 \mathrm{H}, \mathrm{H}-5^{\prime}, \mathrm{H}-6^{\prime}\right.$, H-6"), 2.02, 1.98, 1.93 (3 s, 9 H, 3 OAc), 1.71 (s, $3 \mathrm{H}$, $\mathrm{NH} \underline{\mathrm{Ac}}$ ). Found: $\mathrm{C}$ 41.32; $\mathrm{H} 4.12 ; \mathrm{N} \mathrm{8.19}$. Calc. for $\mathrm{C}_{18} \mathrm{H}_{22} \mathrm{BrN}_{3} \mathrm{O}_{10}$ : C 41.55; $\mathrm{H} 4.26$; $\mathrm{N}$ 8.08. MS: $m / z$ (EI) $520\left(519 / 521\right.$ bromine isotopes) $\left(M^{+}\right)$.

1 - (2-Acetamido-3,4,6-tri-O-acetyl-2-deoxy- $\beta$-D-glucopyranosyl)-5-iodouracil (12). Yield: $0.98 \mathrm{~g}(45 \%) ; R_{\mathrm{f}}$ $\left(\mathrm{CHCl}_{3}-\mathrm{MeOH} \quad 7: 3\right) \quad 0.76$, m.p. $177-181^{\circ} \mathrm{C}$ (from EtOH). UV: $\lambda_{\max }(\mathrm{MeOH}) 279(\log \varepsilon 3.72) .{ }^{1} \mathrm{H}$ NMR: $\delta 7.92(\mathrm{~s}, 1 \mathrm{H}, \mathrm{H}-6), 6.76\left(\mathrm{~s}, \mathrm{H}, \mathrm{N} \underline{H} \mathrm{Ac}, J_{2^{\prime}, \mathrm{NH}} 8.0 \mathrm{~Hz}\right)$, $5.40\left(\mathrm{~d}, 1 \mathrm{H}, \mathrm{H}-1^{\prime}, J_{1^{\prime}, 2^{\prime}} 10.0 \mathrm{~Hz}\right), 5.27\left(\mathrm{t}, 1 \mathrm{H}, \mathrm{H}-3^{\prime}\right.$, $\left.J_{3^{\prime}, 4^{\prime}} 10.0 \mathrm{~Hz}\right), 5.14\left(\mathrm{t}, 1 \mathrm{H}, \mathrm{H}-4^{\prime}, J_{4^{\prime}, 5^{\prime}} 10.0 \mathrm{~Hz}\right), 4.48(\mathrm{q}$, $\left.1 \mathrm{H}, \mathrm{H}-2^{\prime}, J_{2^{\prime}, 3^{\prime}} 10.0 \mathrm{~Hz}\right), 4.26\left(\mathrm{dd}, 1 \mathrm{H}, \mathrm{H}-6^{\prime}, J_{5^{\prime}, 6^{\prime \prime}}\right.$ $5.0 \mathrm{~Hz}$ ), 4.15 (dd, $\left.1 \mathrm{H}, \mathrm{H}-6^{\prime \prime}, J_{6^{\prime}, 6^{\prime \prime}} 12.0 \mathrm{~Hz}\right), 3.96$ (ddd, $\left.1 \mathrm{H}, \mathrm{H}-5^{\prime}, J_{5^{\prime}, 6^{\prime}} 4.0 \mathrm{~Hz}\right), 2.10,2.08,2.06(3 \mathrm{~s}, 9 \mathrm{H}, 3 \mathrm{OAc})$, 1.94 (s, $3 \mathrm{H}$, NHAc). Found: C 38.01; H 3.86; N 7.52. Calc. for $\mathrm{C}_{18} \mathrm{H}_{22} \mathrm{IN}_{3} \mathrm{O}_{10}$ : C 38.11; $\mathrm{H} 3.91 ; \mathrm{N}$ 7.41. MS: $m / z(\mathrm{FAB}>0) 568(M+\mathrm{H})^{+}$. 
Free nucleosides of 2-acetamido-2-deoxy-D-glucose (7), (9), (11) and (13). General deprotection procedure. A solution of the acylated nucleosides $(6,8,10$ and 12 , $0.35 \mathrm{mmol}$ ) in $0.3 \mathrm{M}$ sodium methoxide solution $(10 \mathrm{ml})$ was stirred at room temperature for $3 \mathrm{~h}$. After neutralization with Dowex resin (IR $84-\mathrm{H}^{+}$form), the solution was filtered and the resin was washed with $\mathrm{MeOH}$. The combined extracts were evaporated and the residue was partitioned between water and ether. The aqueous phase was evaporated to dryness and lyophilized from water. The residue was co-evaporated with silica gel $(1 \mathrm{~g})$ and placed on top of a column of silica gel $(10 \mathrm{~g})$. Elution with a gradient of $\mathrm{MeOH}(0-20 \%)$ in $\mathrm{CHCl}_{3}$ afforded the desired nucleosides.

1-(2-Acetamido-2-deoxy- $\beta$-D-glucopyranosyl) uracil (7). Yield: $120 \mathrm{mg}(78 \%) ; R_{\mathrm{f}}\left(\mathrm{CHCl}_{3}-\mathrm{MeOH} 3: 2\right) \quad 0.10$, m.p. $213-215^{\circ} \mathrm{C}$ decomp. (lit. ${ }^{23} 216-217^{\circ} \mathrm{C}$ decomp.). UV: $\lambda_{\max }(\mathrm{MeOH}) 259(\log \varepsilon 3.94) .{ }^{1} \mathrm{H}$ NMR $: \delta 7.92$ $\left(\mathrm{d}, 1 \mathrm{H}, \mathrm{NHAc}, J_{2^{\prime}, \mathrm{NH}} 8.5 \mathrm{~Hz}\right), 7.10(\mathrm{~d}, 1 \mathrm{H}, \mathrm{H}-6), 5.57$ (d, $\left.1 \mathrm{H}, \mathrm{H}-1^{\prime}, J_{1^{\prime}, 2^{\prime}} 10.0 \mathrm{~Hz}\right), 5.30-5.14(\mathrm{~m}, 3 \mathrm{H}, 3 \mathrm{OH})$, $5.19\left(\mathrm{~d}, 1 \mathrm{H}, \mathrm{H}-5, J_{5,6} 7.6 \mathrm{~Hz}\right), 3.83\left(\mathrm{q}, 1 \mathrm{H}, \mathrm{H}-2^{\prime}, J_{2^{\prime}, 3^{\prime}}\right.$ $10.0 \mathrm{~Hz}), 3.64\left(\mathrm{dd}, 1 \mathrm{H}, \mathrm{H}-3^{\prime}, J_{3^{\prime}, 4^{\prime}} 9.0 \mathrm{~Hz}\right), 3.48-3.39$ (m, H-4', H-5'), 3.29-3.08 (m, 2 H, H-6', H-6"), 1.68 (s, $3 \mathrm{H}$, NHAc). Found: C 45.42; H 5.52; N 13.08. Calc. for $\mathrm{C}_{12} \mathrm{H}_{17} \mathrm{~N}_{3} \mathrm{O}_{7}$ : C 45.71; $\mathrm{H}$ 5.44; $\mathrm{N}$ 13.33. MS: $\mathrm{m} / \mathrm{z}$ $(\mathrm{FAB}>0) 338(M+\mathrm{Na})^{+}$.

1 -(2-Acetamido-2-deoxy- $\beta$-D-glucopyranosyl)-5-fluorouracil (9). Yield: $73 \mathrm{mg}(63 \%) ; R_{\mathrm{f}}\left(\mathrm{CHCl}_{3}-\mathrm{MeOH} 3: 2\right)$ 0.17 , m.p. $210-212^{\circ} \mathrm{C}$ (decomp.). UV: $\lambda_{\max }(\mathrm{MeOH}) 264$ $(\log \varepsilon 3.70) .{ }^{1} \mathrm{H}$ NMR: $\delta 8.05\left(\mathrm{~d}, 1 \mathrm{H}, \mathrm{H}-6, J_{6, \mathrm{~F}} 7.0 \mathrm{~Hz}\right)$, $7.95\left(\mathrm{~d}, 1 \mathrm{H}, \mathrm{NHAc}, J_{2^{\prime}, \mathrm{NH}} 8.6 \mathrm{~Hz}\right), 5.35\left(\mathrm{~d}, 1 \mathrm{H}, \mathrm{H}-1^{\prime}\right.$, $\left.J_{1^{\prime}, 2^{\prime}} 9.7 \mathrm{~Hz}\right), 5.04\left(2 \mathrm{~d}, \mathrm{C}_{2}{ }^{\prime}-\mathrm{OH}, \mathrm{C}_{4}{ }^{\prime}-\mathrm{OH}, J 5.0,6.5 \mathrm{~Hz}\right)$, $4.57\left(\mathrm{t}, 1 \mathrm{H}, \mathrm{C}_{6}{ }^{\prime}-\mathrm{OH}, J 5.5 \mathrm{~Hz}\right), 3.81\left(\mathrm{q}, 1 \mathrm{H}, \mathrm{H}-2^{\prime}\right.$, $\left.J_{2^{\prime}, 3^{\prime}} 9.7 \mathrm{~Hz}\right), 3.70\left(\mathrm{t}, 1 \mathrm{H}, \mathrm{H}-3^{\prime}, J_{3^{\prime}, 4^{\prime}} 9.8 \mathrm{~Hz}\right.$ ), 3.46-3.22 (m, 4 H, H-4', H-5', H-6', H-6"), 1.74 (s, 3 H, NHAc). Found: $\mathrm{C}$ 43.12; $\mathrm{H}$ 4.78; $\mathrm{N}$ 12.48. Calc. for $\mathrm{C}_{12} \mathrm{H}_{16} \mathrm{FN}_{3} \mathrm{O}_{7}: \mathrm{C} 43.25 ; \mathrm{H} 4.84 ; \mathrm{N}$ 12.61. MS: $\mathrm{m} / z$ $(\mathrm{FAB}>0) 356(M+\mathrm{Na})^{+}$.

1-(2-Acetamido-2-deoxy- $\beta$-D-glucopyranosyl)-5-bromouracil (11). (a) From deprotection experiment. Yield: $\left.83 \mathrm{mg} \quad(60 \%), \quad R_{\mathrm{f}} \quad\left(\mathrm{CHCl}_{3}-\mathrm{MeOH}\right) \quad 3: 2\right) \quad 0.18$, m.p. $278-282^{\circ} \mathrm{C}$ (decomp.) (from EtOH-ether). UV: $\lambda_{\max }(\mathrm{MeOH})(268)\left(\log \varepsilon\right.$ 3.70). ${ }^{1} \mathrm{H}$ NMR: $\delta 11.82$ (s, $1 \mathrm{H}, \mathrm{NH}), 8.15$ (d, $1 \mathrm{H}, \mathrm{H}-6), 7.73$ (d, $1 \mathrm{H}$, NHAc, $\left.J_{2^{\prime}, \mathrm{NH}} 8.9 \mathrm{~Hz}\right), 5.39\left(\mathrm{~d}, 1 \mathrm{H}, J_{1^{\prime}, 2^{\prime}} 9.5 \mathrm{~Hz}\right), 4.66-4.41(\mathrm{~m}$, $3 \mathrm{H}, 3 \mathrm{OH}), 3.86\left(\mathrm{q}, 1 \mathrm{H}, \mathrm{H}-2^{\prime}, J_{2^{\prime}, 3^{\prime}} 10.0 \mathrm{~Hz}\right), 3.85(\mathrm{t}$, $\left.1 \mathrm{H}, \mathrm{H}-3^{\prime}, J_{3^{\prime}, 4^{\prime}} 10.0 \mathrm{~Hz}\right), 3.49-3.37$ (m, $\left.2 \mathrm{H}, \mathrm{H}-4^{\prime}, \mathrm{H}-5^{\prime}\right)$, 3.29-3.05 (m, 2 H, H-6', H-6"), 1.72 (s, 3 H, NHAc). Found: $\mathrm{C}$ 36.69; $\mathrm{H}$ 4.13; $\mathrm{N}$ 10.42. Calc. for $\mathrm{C}_{12} \mathrm{H}_{16} \mathrm{BrN}_{3} \mathrm{O}_{7}$ : C 36.57; $\mathrm{H} 14.09 ; \mathrm{N}$ 10.66. MS: $\mathrm{m} / \mathrm{z}$ (EI) 394 (393/395 bromine isotopes) $\left(M^{+}\right)$.

(b) From reaction of 7 with $\mathrm{N}$-bromosuccinimide. $N$ Bromosuccinimde (NBS, $0.20 \mathrm{~g}, 1.1 \mathrm{mmol}$ ) was added at $35^{\circ} \mathrm{C}$ to a suspension prepared by mixing a solution of $7(0.31 \mathrm{~g}, 1.0 \mathrm{mmol})$ in 1,2-dimethoxyethane $(25 \mathrm{ml})$ and DMF $(5 \mathrm{ml})$ with a solution of sodium azide $(0.26 \mathrm{~g}$, $4.0 \mathrm{mmol})$ in water $(1 \mathrm{ml})$. The reaction mixture was stirred for $3 \mathrm{~h}$ at $35^{\circ} \mathrm{C}$, after which the solvent was removed in vacuo and the residue was purified by silica gel chromatography using a gradient of $\mathrm{MeOH}(0-20 \%)$ in $\mathrm{CHCl}_{3}$ to give $11(0.22 \mathrm{~g}, 56 \%)$ as a solid. The ${ }^{1} \mathrm{H}$ NMR and mass spectra, m.p. and mixed m.p. were identical with those for the compound prepared in experiment (a).

1-(2-Acetamido-2-deoxy- $\beta$-D-glucopyranosyl)-5-iodouracil (13). (a) From deprotection experiment. Yield: $153 \mathrm{mg}$ (77\%); $R_{\mathrm{f}}\left(\mathrm{CHCl}_{3}-\mathrm{MeOH} 7: 3\right) 0.30$, m.p. $185-188^{\circ} \mathrm{C}$ (decomp.). UV: $\lambda_{\max }(\mathrm{MeOH}) 275$ (log $\left.\varepsilon 3.80\right) .{ }^{1} \mathrm{H}$ NMR: $\delta 7.98$ (s, $1 \mathrm{H}, \mathrm{H}-6), 7.94$ (d, $1 \mathrm{H}, \mathrm{NHAc}, J_{2^{\prime}, \mathrm{NH}}$ $10.0 \mathrm{~Hz}), 5.38\left(\mathrm{~d}, 1 \mathrm{H}, \mathrm{H}-1^{\prime}, J_{1^{\prime}, 2^{\prime}} 9.5 \mathrm{~Hz}\right), 3.90(\mathrm{q}, 1 \mathrm{H}$, $\left.\mathrm{H}-2^{\prime}, J_{2^{\prime}, 3^{\prime}} 9.5 \mathrm{~Hz}\right), 3.71-3.25$ (m, 8 H, H-3', H-4', H-5', H-6', H-6", $3 \mathrm{OH}$ ), 1.71 (s, $3 \mathrm{H}, \mathrm{NHAc}$ ). Found: C 32.31; $\mathrm{H}$ 3.51; $\mathrm{N}$ 9.42. Calc. for $\mathrm{C}_{12} \mathrm{H}_{16} \mathrm{IN}_{3} \mathrm{O}_{7}: \mathrm{C} 32.67 ; \mathrm{H}$ 3.66; N 9.52. MS: $m / z(\mathrm{FAB}>0) 442(M+\mathrm{H})^{+}, 464$ $(M+\mathrm{Na})^{+}$.

(b) From reaction of 7 with iodine monochloride. Iodine monochloride ( $\mathrm{ICl}, 0.40 \mathrm{~g}, 2.5 \mathrm{mmol}$ ) was added slowly over a $5 \mathrm{~min}$ period to a suspension of sodium azide $(0.26 \mathrm{~g}, 4.0 \mathrm{mmol})$ in acetonitrile $(30 \mathrm{ml})$ at ice-bath temperature and the stirring was continued for another $5 \mathrm{~min}$. To this, a solution of $7(0.31 \mathrm{~g}, 1.0 \mathrm{mmol})$ in acetonitrile $(30 \mathrm{ml})$ and $\mathrm{DMF}(3 \mathrm{ml})$ was added and the reaction mixture was warmed to $25^{\circ} \mathrm{C}$ and stirred for $24 \mathrm{~h}$. The solvent was evaporated off and the residue was poured onto a column of silica gel. Elution with a gradient of $\mathrm{MeOH}(0-20 \%)$ in $\mathrm{CHCl}_{3}$ afforded $13(0.22 \mathrm{~g}$, $50 \%$ ). The ${ }^{1} \mathrm{H}$ NMR and mass spectra, m.p. and mixed m.p. were identical with those for the compound prepared in experiment (a).

Acknowledgements. One of the authors (F. B. Issa) would like to thank the Basrah Institute of Technology for a scholarship. We thank Mrs. W. B. Böer, Mr. K. Hägele and Mrs. M. J. Quelle of Fakultät für Chemie, Universität Konstanz (Germany) for the elemental analyses and the mass spectra.

\section{References}

1. Robins, R. K. Chem. Eng. News 64 (1986) 28

2. (a) Stetson, P. L., Normolle, D. P., Knol, J. A., Johnson, N. J., Yang, Z., Sakmar, E., Prieskorn, D., Terrio, P. Knutsen, C. A. and Ensminger, W. D. J. Natl. Cancer Inst. 83 (1991) 1659; (b) Davies, D. B. In: Emsley, J. W., Feeney, J and Sutcliffe, L. H., Eds., Progress in Nuclear Magnetic Resonance Spectroscopy, Pergamon Press, New York 1978, Vol. 12, pp. 135-225; (c) Goodman, L. In: Ts'O, O. P., Ed., Basic Principles in Nucleic Acid Chemistry, Academic Press, New York 1974, pp. 146-152; (d) Uesugi, S. and Ikehara, M. Chem. Pharm. Bull. 26 (1978) 3040; (e) Harbers, E., Chaudhuri, N. K. and Heidelberger, C. J. Biol. Chem. 234 (1959) 1255; (f) Prussof, W. H. Cancer Res. 20 (1960) 92; (g) Zamenhof, S., 
Reiner, B., De Giovanni, R. and Rich, K. J. Biol. Chem. 219 (1956) 165; (h) Dunn, D. B. and Smith, J. D. Biochem. J. 67 (1957) 494.

3. (a) De Clercq, E., Balzarini, J., Torrence, P. F., Mertes, M. P., Schimdt, C. L., Shugar, D., Barr, P. J., Jones, A. S., Verhelst, G. and Walker, R. T. Mol. Pharmacol. 19 (1981) 321; (b) De Clercq, E., Descamps, J., Verhelst, G., Walker, R. T., Jones, A. S., Torrence, P. F. and Shugar, D. J. Infect. Dis. 141 (1980) 563; (c) Prusoff, W.H. and Fischer, P. H. In: Walker, R. T., De Clercq, E. and Eckstein F., Eds., Nucleoside Analogues: Chemistry and Medical Applications, 1979, Vol. 26, pp. 281-318; (d) Lin, T. S., Chen, M. S., McLaren, C., Gao, Y. S. Ghazzouli, I. and Prussof, W. H. J. Med. Chem. 30 (1987) 440; (e) De Clercq, E. Arch. Int. Physiol. Biochim. (1979) 353.

4. Aerschot, A. V., Everaet, D., Balzarini, J., Augustyns, K., Jie, L., Janssen, G., Peeters, O., Balton, N., De Ranter, C., De Clercq, E. and Herdewjin, P. J. Med. Chem. 33 (1990) 1833.

5. Bretner, M., Kulikowski, T., Dzik, J. M., Balinska, M., Rode, W. and Shugar, D. J. Med. Chem. 36 (1993) 3611.

6. Danenberg, P. V. Biochim. Biophys. Acta 473 (1977) 73.

7. Heidelberger, C., Danenberg, P. V. and Moran, R. G. Adv. Enzymol. 54 (1983) 57.

8. Bretner, M., Balinska, M., Krawiec, K., Kierdaszuk, B., Shugar, D. and Kulikowski, T. Nucleosides Nucleotides 14 (1995) 657.

9. Kulikowski, T.. Bretner. M.. Dzik, J. M.. Zielinski, Z., Ciesle, J., Rode, W., Vilpo, J. A. and Shugar, D. Nucl. Acid Res. Symp. Ser. 18 (1987) 61.

10. Harris, A., Balagopala, M. I. and Ollapally, A. P. 11th. Int. Round Table on Nucleosides and Nucleotides and their Biological Applications, Leuven, Belgium, Sept. 7-11, 1994, p. 76.

11. Prusoff, W. H., Holmes, W. L. and Welch, A. D. Cancer Res. 13 (1953) 221.
12. Awano, H., Shutto, S., Baba, M., Kira, T., Shigeta, S. and Matsuda, A. Bioorg. Med. Chem. Lett. 4 (1994) 367.

13. Kumar, R., Wiebe, L. I., Knaus, E. E., Allen, T. M. and Tempest, M. L. Drug Des. Discovery 8 (1992) 179.

14. Kobayashi, Y., Kumadaki, I. and Yamamato, K. J. Chem. Soc., Chem. Commun. (1977) 536.

15. Iwashina, T., Tovell, D. R., Xu, L., Tyrrell, D. L., Knaus, E. E. and Wiebe, L. I. Drug Des. Delivery 3 (1988) 309.

16. Bradschaw, T. K. and Hutchinson, D. W. Chem. Soc. Rev. 6 (1977) 43.

17. Long, R. A., Robins, R. K. and Towsend, L. B. J. Org. Chem. 32 (1967) 2751.

18. Rahim, S. G., Duggan, M. J. H., Walker, R. T., Jones, A. S., Dyer, R. L., Balzarini, J. and De Clercq, E. Nucleic Acid Res. 10 (1982) 5285.

19. Coffy, S. Rodds' Chemistry of Carbon Compounds, Aliphatic Compounds, Elsevier, Amsterdam, 1967, Vol. 1, Part F, p. 483.

20. Martin, D. W., Mayer, P. A., Rodwell, V. W. and Granner, D. K. Harpers' Reviews of Biochemistry, 20th. ed. Lang Medical Publication, CA 1985, p. 150.

21. Strange, R. E. and Powell, J. F. Biochem. J. 58 (1954) 80.

22. Gordon, C. Antineoplastic and Immunosuppressive Agents, Springer, Berlin 1974, Part 1, Chap. 1.

23. Stevens, C. L. and Nagarajan, K. J. Am. Chem. Soc. 5 (1962) 1124

24. Hilbert. G. E. and Johnson. T. B. J. Am. Chem. Soc. 52 (1930) 4489.

25. Birkofer, L. and Ritter, A. Angew. Chem. 77 (1965) 414.

26. Vorbrüggen, H. and Höfle, G. Chem. Ber. 114 (1981) 1256.

27. Kumar, R., Wiebe, L. I. and Knaus, E. E. Can. J. Chem. 72 (1994) 2005.

28. Al-Masoudi, N. A. and Pfleiderer, W. Tetrahedron 49 (1993) 7579.

Received December 27, 1996. 